\title{
FIGHT AGAINST SUPERBUGS IN A BURN CENTRE: ARE WE DOING ENOUGH
}

\author{
Muhammad Rizwan Aslam, Taokeer Ahmed Rizvi, Muhammad Tariq Munawar, Muhamad Ashraf Hussain, Tabish Samuel* \\ Combined Military Hospital Kharian/National University of Medical Sciences (NUMS) Pakistan, *Combined Military Hospital /National University of Medical \\ Sciences (NUMS) Rawalpindi Pakistan
}

\section{ABSTRACT}

Objective: To determine the superbug infection in a burn centre, its impact on mortality/morbidity and to review all preventive/therapeutic steps taken to fight this menace.

Study Design: Retrospective cross sectional study.

Place and Duration of Study: Department of Burns \& Plastic Surgery, Army Burn Centre, Combined Military Hospital Kharian, from Oct 2018 to Sep 2019.

Methodology: A detailed retrospective audit of departmental data was carried out. Parameters like direct admission vs transferred patient, percentage of burns (Total Burn Surface Area-TBSA\%), records of all burns related deaths and all culture / sensitivity reports were analysed using SPSS-20.

As a standard practice in our unit blood, tracheal secretions and pus culture specimens of all patients are collected at the time of admission and then periodically fresh samples are taken every week or earlier when-ever required.

Results: Out of 515 patients, $283(54.95 \%)$ were children under the age of 12 years. The overall survival rate improved by $13.43 \%$ as compared to last year. Out of 584 bacteriology reports 396 (67.81\%) were positive and $188(32.19 \%)$ were negative. On culture 508 organisms were isolated, majority of which were Carbapenem Resistant Pseudomonas, Acinetobacter, Enterobacteriaceae and Methicillin resistant staphylococcus aureus.

Conclusion: Multi drug resistant superbug infection is a worldwide menace. The best clinical practices, strict contact isolation, enhanced environmental cleaning and judicious use of appropriate antibiotics are the main strategies in this war. Need for newer more effective antibiotics cannot be overemphasized.

Keywords: Burn, Carbapenem resistant, Mortality, Multi drug resistant, Sepsis, Superbug.

This is an Open Access article distributed under the terms of the Creative Commons Attribution License (https://creativecommons.org/licenses/by-nc/4.0/), which permits unrestricted use, distribution, and reproduction in any medium, provided the original work is properly cited.

\section{INTRODUCTION}

The first true antibiotic, was discovered by Alexander Fleming, Professor of Bacteriology at St. Mary's Hospital in London in 1928. No new classes of antibiotics have been discovered since the $1980 \mathrm{~s}^{1}$. The antibiotics that have been brought to market in the past three decades are variations of drugs that have been discovered before ${ }^{2}$. Bacteria are highly adaptable organisms which have an extraordinary ability to mutate in response to their environmental conditions. Number of bacterial organisms decipher how to resist the drug's bactericidal effects. These bacteria develop genome mutations or resistance genes. These bacteria multiply to produce a population of antibiotic-resistant organisms. These resistant bacteria transfer their newly acquired resistance genes to other species of bacteria through the process of conjugation (a reproductive interaction) $)^{3,4}$. This transfer of resistance to other bacterial species give rise to new strains that have been termed superbugs which resist the effects of existing antibiotics. Decades of overuse and misuse of antibiotics have caused this crisis. The next major global pandemic may

Correspondence: Dr Muhammad Rizwan Aslam, Consultant Burn \& Plastic Surgeon, CMH Kharian Pakistan

Received: 13 May 2020; revised received: 29 Sep 2020; accepted: 14 Oct 2020 involve an antibiotic-resistant superbug 5 .

Burn centres worldwide are also facing this challenge because significant thermal injury induces a state of immunosuppression ${ }^{6}$, that predisposes burn patient to overwhelming sepsis and high mortality, this is further compounded by loss of skin barrier the biggest protection against these bugs. As per CDC data they have identified 6 strains of superbugs that are causing life threatening sepsis in ICU's worldwide, table-I.

To prevent spreading MDR superbugs, the CDC recommends use of, contact isolation precautions, enhanced environmental cleaning, dedicated patient care equipment and prudent use of antibiotics ${ }^{7}$. We are following strict contact isolation protocols. All staff is regularly monitored and trained in hand hygiene and barrier nursing techniques. Enhanced environmental cleaning is being ensured by strictly following the protocols of our centre. Acute ICU area has digital lock and limited access to authorized personnel only. Acute ICU area has HVAC system with HEPA filters installed. Rooms are fumigated with formalin/H2O2 \& Silver vapour. Room is sealed for 12 to $24 \mathrm{hrs}$, after every patient moving out. Bedding is changed daily before 8 am. Every room has two lidded bins separate color- 
coded for biohazardous and routine waste. Thrice daily dustbins are emptied in each room. With every new patient complete bed including mattress is changed (previous bed is sent to sanitization bay for washing, spraying and sunlight treatment). Only one attendant is allowed who wears mask and cap with gloves all the time. Only patient is fed inside room, no fruit or food item is allowed to be kept in patient room.

All ventilator disposables are discarded, machine and filters are regularly changed and cleaned with every patient. Every room has mask, gloves and alcohol-based hand sanitizer installed outside the door. Previously 2 but now we have $4 \times$ Operation theatres, One OT is daily closed after fumigation for $24 \mathrm{hrs}$ and three remain functional. Laminar flow air with HVAC system \& HEPA filters are installed. Every operation table has a foot end water trap draining into floor trap/receptacle. Every patient is bathed inside operation theatres with 5-20 gallons of Zero tedious RO water. Sterilized Macintosh sheet is changed with each patient. Zero tedious water is used for patient bathing and all staff washing/CSSD. Strict CSSD protocols are followed in our own dedicated CSSD. Hand and nasal swabs of staff are taken after every 3 months. Surface swabbing of critical areas is also done periodically.

We use Filgrastim (Colony stimulating factor) for all cases with neutropenia. All major burns are given Propranolol (Inderal) $1-2 \mathrm{mg} / \mathrm{Kg}$ body weight. The Albumin of patient is kept above $30 \mathrm{gm}$, all patients are given Human Albumin as \& when required. Immunoglobins (IVIG, BISEKO \& Pentaglobin) are used judiciously where required.

\section{METHODOLOGY}

This retrospective cross sectional study was conducted at department of Burns \& Plastic surgery, Army Burn Centre, Combined Military Hospital Kharian, from October 2018 to September 2019.

All acute burn cases irrespective of age/gender or severity were included in the study whereas all old burns and burn cases requiring secondary surgeries for previously treated burns were excluded from the study. Written permission was obtained from hospital research ethics committee ensuring patient's confidentiality vide (IRB/13/Khn/2019). Burns were tabulated into TBSA \% groups and mortality compared in each group.

Bacteriology was not done in non-infected patients with superficial burns that showed intent to heal uneventfully without any major surgical interventions.
Blood, tissue/surface, CVP tips, Foley catheter tips, tracheal secretions/sputum and urine/stool samples were sent for bacteriological examination. Our standard policy is taking blood, tracheal secretions and pus culture specimens of all patients at the time of admission and then periodically take fresh samples every week or earlier when-ever required.

At admission, all patients were given two antibiotics with standard recommended dosage as per age and creatinine clearance. The dosage and type of antibiotics were changed/adjusted after evaluating patient's response, general condition, presence of fever and other signs of infection. Data was analysed using Statistical Package for Social Sciences (SPSS) version 20. Descriptive statistics were applied to find frequencies, percentages, means, and standard deviations. Quantitative variables, such as age and TBSA\%, were expressed as means and standard deviations. Qualitative variable, such as type of microorganism, was expressed as frequencies and percentages.

\section{RESULTS}

A total of 515 patients fulfilling the inclusion and exclusion criteria were included in our study. Their age ranged from 25 days to 73 years (mean $23 \pm 12.2$ years). There were $295(57.28 \%)$ male and $220(42.78 \%)$ females, male to female ratio was 1.3:1. Children under 12 years of age were $283(54.95 \%)$ and adults $232(45.05 \%)$. There were $294(57.09 \%)$ direct admissions and 221 $(42.91 \%)$ indirect admissions, who were initially treated at home, by quacks or other hospitals and most of the time brought due to complications/sepsis.

Table-I: Superbug strains in present day intensive care unit's.

Carbapenem-resistant Enterobacteriaceae (CRE) (E. coli, Klebsiella pneumoniae, Providencia, proteus)

Methicillin-resistant Staphylococcus aureus (MRSA)

ESBL-producing Enterobacteriaceae (extended-spectrum $\beta$-lactamases)

Vancomycin-resistant Enterococcus (VRE)

Carbapenum-resistant Pseudomonas aeruginosa (CRPA) Carbapenum-resistant Acinetobacter Baumannii (CRAB)

${ }^{*}$ Source CDC official website

TBSA \% (Total body surface area percent) of burns ranged from $8 \%$ (full thickness) to $95 \%$ mixed thickness burns. All the burns were tabulated in to TBSA\% groups and mortality compared in each group, table-II.

Comparison of data between Oct 2017-Sept 2018 and October 2018-Sept 2019 reveals that there was an overall improvement in survival rate by $13.43 \%$ in year 
2018-2019 due to our improved protocols and procedures as explained in introduction.

Bacteriology was not required in $60(11.65 \%)$ noninfected patients with superficial burns that healed uneventfully without any major surgical interventions, whereas in $455(88.35 \%)$ patients one or more bacteriology samples were sent. teriaceae group) were 113 (22.24\%), MRSA (methicillin resistant staphylococcus aureus) 86 (16.93\%) and CRAB (carbapenem resistant Acinetobacter Baumannii) were $63(12.40 \%)$ besides other lesser virulent strains, table-III.

The most worrisome and serious condition was a synergistic infection with two or more organisms. We

Table-II: Mortality comparison with previous year.

\begin{tabular}{|c|c|c|c|c|c|c|c|}
\hline \multirow{3}{*}{$\begin{array}{l}\text { Total Body Surface } \\
\text { Area Burn Percentage } \\
\text { TBSA \% }\end{array}$} & \multicolumn{6}{|c|}{ Mortality } & \multirow{3}{*}{$\begin{array}{c}\text { Mortality Ratio } \\
\text { Percentage }\end{array}$} \\
\hline & \multicolumn{3}{|c|}{ Oct 2017-Sept 2018} & \multicolumn{3}{|c|}{ Oct 2018 - Sept 2019} & \\
\hline & Total & Death & $\%$ & Total & Death & $\%$ & \\
\hline $1-24$ & 170 & 5 & 3.25 & 245 & 8 & 3.27 & +0.02 \\
\hline $25-34$ & 68 & 13 & 19.12 & 99 & 15 & 15.15 & -3.97 \\
\hline $35-44$ & 82 & 29 & 35.37 & 69 & 24 & 34.78 & -0.59 \\
\hline $45-54$ & 41 & 28 & 68.29 & 33 & 18 & 54.55 & -13.74 \\
\hline $55-64$ & 24 & 22 & 91.67 & 19 & 15 & 78.95 & -12.72 \\
\hline $65-74$ & 33 & 28 & 84.85 & 12 & 11 & 99.67 & +14.82 \\
\hline $75-90$ & 36 & 34 & 96.80 & 20 & 19 & 95.00 & -1.80 \\
\hline Total & 457 & 159 & 34.79 & 515 & 110 & 21.36 & +13.34 \\
\hline
\end{tabular}

Total 584 samples were sent for bacteriological examination, which included 208 (35.62\%) Blood, 251 (42.98\%) tissue/surface, 56 (9.59\%) CVP tips, 29 (4.97\%) Foley catheter tips, $22(3.77 \%)$ tracheal secretions/sputum and 18 (3.08\%) urine/stool samples, 188 (32.19\%) samples yielded no growth and were marked negative whereas 396 (67.81\%) reports were positive. Total 508 microorganisms were grown and their sensitivity checked.

Majority of isolates were superbugs; CRPA (carbapenem resistant pseudomonas aeruginosa) were 166 (32.68\%), MDR (Multidrug resistant)/XDR (extensively drug resistant) CRE (carbapenem resistant Enterobac-

Table-III: Microorganisms isolated.

\begin{tabular}{l|c}
\hline Organism & $\begin{array}{c}\text { Positive } \\
\text { Tests (\%) }\end{array}$ \\
\hline MDR Pseudomonas Aeruginosa (CRPA) & $166(32.67)$ \\
\hline MDR Acinetobacter Baumannii (CRAB) & $63(12.4)$ \\
\hline MDR Staphlococcus Aureus (MRSA) & $86(16.92)$ \\
\hline Klebsiella Pneumonae (CRE) & $65(12.79)$ \\
\hline Enterococcus species & $21(4.13)$ \\
\hline Citrobcter Freundii & $6(1.18)$ \\
\hline Strentrophomonas Maltophilia & $11(2.17)$ \\
\hline Providentia Stuarti (CRE) & $15(2.95)$ \\
\hline Serratia Marcescens & $9(1.77)$ \\
\hline E. Coli (CRE) & $18(3.55)$ \\
\hline Enterobacter Cloacae & $6(0.12)$ \\
\hline Coagulase negative staph species (CONS) & $27(5.31)$ \\
\hline Proteus Mirabilis (CRE) & $15(2.95)$ \\
\hline Total organisms isolated & $508(100)$ \\
\hline
\end{tabular}

observed CRPA/CRAB with a CRE or MRSA as being the most lethal combinations. In 29 (6.37\%) patients there were two concomitant serious isolates (CRPA + CRAB) and in 18 (3.96\%) patients three superbugs (CRPA+CRAB+MRSA) were isolated. $65 \%$ of these cases did not survive. It was also noted that $85 \%$ of these cases were indirect admissions transferred from other hospitals with established sepsis at the time of admission. The drug resistance rate of different organisms is given in table-IV.

It is clearly evident that both Pseudomonas and Acinetobacter are $86 \%$ and $92 \%$ resistant to carbapenems and $88-98 \%$ resistant to quinolones, aminoglycosides and pipracillin/Tazobactum respectively. Staph Aureus showed 34\% resistance to fusidic acid and 22\% resistance to Clindamycin, but was $100 \%$ sensitive to Vancomycin and $94 \%$ to Linezolid. The carbapenem resistant Enterobacteriaceae group in our study only showed intermediate resistance to carbapenems about $34-60 \%{ }^{25}$.

There were three outbreaks of these polymicrobial infections during this period of study. In all three episodes we traced back the infection to have come from other burn units/ICU of tertiary care hospitals. Each episode lasted from 1-2 weeks. These bugs are brought by one patient and they end up infecting 3-7 patients admitted at that time, all serious immunocompromised major burns cases. Each time partial closure of burn centre and other extensive measures were taken to eradicate these infections. 
Table-IV: Drug resistance pattern among different MDR/XDR organisms.

\begin{tabular}{|c|c|c|c|c|c|}
\hline \multirow{2}{*}{ Drug } & \multicolumn{5}{|c|}{ \% Age Resistance } \\
\hline & P. Aeruginosa & S. Aureus & A. Baumannii & K. Pneumonae & E. Coli \\
\hline Meropenem & 88 & - & 92 & 70 & 40 \\
\hline Imipenem & 86 & - & 92 & 70 & 40 \\
\hline Ciprofloxain & 94 & 86 & 98 & 92 & 82 \\
\hline Levofloxacin & 94 & - & 98 & 84 & 82 \\
\hline Cetazidime & 94 & - & 96 & 96 & 60 \\
\hline Cefepime & 84 & - & 96 & 92 & 60 \\
\hline Amikacin & 78 & 14 & 84 & 46 & 20 \\
\hline Clindamycin & - & 22 & - & - & - \\
\hline Tazobactm/piperacillin & 88 & - & 94 & 84 & 84 \\
\hline Tigecycline & NT & - & 46 & 24 & 24 \\
\hline Polymyxin B & 02 & 00 & 00 & 00 & 00 \\
\hline Colistin & 02 & 00 & 00 & 00 & 00 \\
\hline Linezolid & - & 06 & - & - & - \\
\hline Fusidic acid & - & 34 & - & - & - \\
\hline Vancomycin & - & 00 & - & - & - \\
\hline
\end{tabular}

\section{DISCUSSION}

Multiple drug resistant and carbapenem resistant strains of bacteria are becoming a true menace and challenge for every intensive care setting in health care systems ${ }^{8}$. More and more of these, hospital acquired superbug infections are causing ever increasing rate of mortality. Millions of people are infected with these super bugs worldwide and almost $20-30 \%$ of them die in spite of best possible care ${ }^{9}$. Prevention and timely treatment of these MDR infections is a challenge. The fight against these superbugs is multifaceted and complex.

Fight against superbug (MDR strain) infections is a daunting task, faced by all major ICU's and burn centre worldwide ${ }^{10,11}$. The findings of our study were more or less similar to national and international literature. In our centre majority of fatal/serious infections were due to CRPA, CRAB, CRE and MRSA as is evident from $\mathrm{WHO}$ and $\mathrm{CDC}$ listing. A similar study in a burn centre in a neighbouring city by Chaudhary et al ${ }^{11}$, also showed a very similar prevalence of MDR superbugs with CRPA/MRSA/CRAB/CRE to be in the same order of prevalence. Although their sample size was much smaller than ours 109 vs 515 patients and 158 vs 508 isolated organisms. CRE are nightmare bacteria, they pose a triple threat ${ }^{19}$. First, they are resistant to all or nearly all antibiotics, even some of our last-resort drugs. Second, they have high mortality rates and kill up to half of people who get serious infections with them. Third, they can spread their resistance to other bacteria ${ }^{5,6,12}$.

A comprehensive literature review presented by Khan et al13, from Malaysia studied the incidence and risk of MRSA infection in burn centres. He concluded that there was a $55 \%$ risk of MRSA infection in a burn ICU, which is much more than our observation. Prevalence in our centre of MRSA is 17\% rather the most common is MDR strain of pseudomonas. So far like their observation the strain prevalent is also sensitive to vancomycin and teicoplanin. Our observation of $>85-98 \%$ resistance of Pseudomonas, Acinetobacter and Klebsiella to carbapenems, quinolones and aminoglycosides is also proven by a recent research of Gupta et al, from India ${ }^{11}$.

One of the main cause of this worldwide MDR superbug menace is injudicious use of antibiotics ${ }^{9,14,15}$. But fact of the matter is that the main armamentarium against this menace, besides adhering to all protocols relating to patient/environment and medical management is antibiotics.

We have our own institutional antibiogram ${ }^{16-18}$, we strive for scientifically judicious usage of appropriate antibiotics as per our community/hospital bug prevalence. The $13.43 \%$ improvement in overall survival rate as compared to last year is a testament to all the policies and procedures that we are strictly adhering to, in the light of CDC guidelines. The main impact on improvement in survival is seen in group from $45-65 \%$ serious burns. It was observed that there were more paediatric burns admitted this year $(54.95 \%)$. The cause of this is that we receive lot of referral/transfers from other hospitals. There is no dedicated paediatric burn care facility in whole country, besides poverty/ crammed up living and social distractions are causing more and more careless paediatric accidents mostly scalds and immersions. 
The last line of defense against these superbugs are polymyxins/Colistin (table-IV) ${ }^{1,2}$. Carbapenemresistant Acinetobacter baumannii (CRAB) $)^{4,16,19,20}$, is a perilous nosocomial pathogen causing substantial morbidity and mortality worldwide. Current treatment options for CRAB are limited and suffer from pharmacokinetic limitations, such as high toxicity and low plasma levels ${ }^{21-23}$. There is alarming rise of carbapenem resistance. Management of Carbapenem resistant organism infections is a challenge.

Two groups of polymyxins are currently being used in clinical practice; polymyxin B and polymyxin $\mathrm{E}$ (otherwise called as colistin). Polymyxin B is not marketed in Pakistan. The therapeutic challenge includes high nephrotoxicity and neurotoxicity, difficulty in Optimizing dosage, promotion of resistance during sub-optimal dosage, lack of universal harmonization of dosing units with respect to critically ill individuals and narrow therapeutic window and low mutant prevention concentration. The only drug of this group available in Pakistan is Polymyxin E (brand name Colistim 2M units/vial). Therapeutic dose is 2 Million units I/ V $\times 8$ hourly. It is fairly ineffective as monotherapy now. Combination therapy with Minocycline, Tigecycline, Chloramphenicol and Fosfomycin are being suggested by extended range sensitivity screening ${ }^{24,25}$. Novel drugs like Cefiderocol, Eravacycline, Plazomicin are under trials but not freely available for clinical use $^{18}$.

We will have to keep on changing our tactics and strictly adhere to prevention protocols to save our patients from these superbugs which are becoming so widely spread in our communities.

\section{CONCLUSION}

MDR superbug infection is a worldwide menace. The best clinical practices, strict contact isolation, enhanced environmental cleaning and judicious use of appropriate antibiotics are the main strategies in this war. Need for newer more effective antibiotics cannot be overemphasized.

\section{CONFLICT OF INTEREST}

This study has no conflict of interest to be declared by any author.

\section{REFERENCES}

1. Velkov T, Roberts KD, Nation RL, Thompson PE, Li J. Pharmacology of polymyxins: new insights into an 'old' class of antibiotics. Future Microbiol 2013; 8(6): 711-24.

2. Balkhair A, Al-Muharrmi Z, Al'Adawi B, Al Busaidi I, Taher HB, Al-Siyabi T, et al. Prevalence and 30-day all-cause mortality of carbapenem-and colistin-resistant bacteraemia caused by Acine- tobacter baumannii, Pseudomonas aeruginosa, and Klebsiella pneumoniae: Description of a decade-long trend. Int J Infect Dis 2019; 85(1): 10-15.

3. Douglas MW, Mulholland K, Denyer V, Gottlieb T. Multi-drug resistant Pseudomonas aeruginosa outbreak in a burn unit-an infection control study. Burns. 2001; 27(2): 131-35.

4. Roca I, Paula AE, Xavier V and Jordi V. The acinetobacter baumannii oxymoron: commensal hospital dweller turned pandrug-resistant menace. Front Microbio 2012 23(3): 148-52.

5. Sousa D, Ceniceros A, Galeiras R, Pértega-Díaz S, GutiérrezUrbón JM, Rodríguez-Mayo M. Microbiology in burns patients with blood stream infections: trends over time and during the course of hospitalization. Infect Dis (Lond) 2018; 50(4): 289-96.

6. Bar-Yoseph H, Cohen N, Korytny A, Andrawus ER, Dar RE, Geffen Y, et al. Risk factors for mortality among carbapenemresistant enterobacteriaceae carriers with focus on immunosuppression. J Infect 2019; 78(2): 101-105.

7. Gupta M, Naik AK, Singh SK. Bacteriological profile and antimicrobial resistance patterns of burn wound infections in a tertiary care hospital. Heliyon 2019; 5(12): e02956-59.

8. Potron A, Poirel L, Nordmann P. Emerging broad-spectrum resistance in Pseudomonas aeruginosa and Acinetobacter baumannii: Mechanisms and epidemiology. Int J Antimicrob Agents 2015 45(6): 568-85.

9. Nayak N, Lenka RK, Padhy RN. Surveillance of multidrug resistant suppurative infection causing bacteria in hospitalized patients in an Indian tertiary care hospital. J Acute Dis 2014; 3(2): 148-56.

10. Singh NP, Rani M, Gupta K, Sagar T, Kaur IR. Changing trends in antimicrobial susceptibility pattern of bacterial isolates in a burn unit. Burns. 2017; 43(5): 1083-87.

11. Chaudhary NA, Munawar MD, Khan MT, Rehan K, Sadiq A. Epidemiology, bacteriological profile, and antibiotic sensitivity pattern of burn wounds in the burn unit of a tertiary care hospital. Cureus 2019; 11(6): 1-5.

12. Senchyna F, Gaur RL, Sandlund J, Truong C, Tremintin G, Kültz $D$, et al. Diversity of resistance mechanisms in carbapenem-resistant Enterobacteriaceae at a health care system in Northern California, from 2013 to 2016. Diagn Microbiol Infect Dis 2019; 93(3): 250-57.

13. Khan TM, Kok YL, Bukhsh A, Lee LH, Chan KG, Goh BH. Incidence of methicillin resistant Staphylococcus aureus (MRSA) in burn intensive care unit: a systematic review. Germs 2018; 8(3): 113-16.

14. Jahangir SU, Rehman M, Munir MK. Identification and Drug Susceptibility Pattern of Pathogenic Bacterial Species among Burn Patients. Pak J Med Health Sci 2017; 11(1): 698-702.

15. De Rosa A, Mutters NT, Mastroianni CM, Kaiser SJ, Günther F Distribution of carbapenem resistance mechanisms in clinical isolates of XDR Pseudomonas aeruginosa. Eur J Clin Microbiol Infect Dis 2019; 38(8): 1547-52.

16. Lowings M, Ehlers MM, Kock MM. Acinetobater baumannii: a superbug. The battle against microbial pathogens: basic science, technological advances and educational programs. $1^{\text {st }}$ ed: Badajoz: Formatex Research Center 2015: 6(2): 587-97.

17. Moghadam SO, Pourmand MR, Aminharati F. Biofilm formation and antimicrobial resistance in methicillin-resistant Staphylococcus aureus isolated from burn patients, Iran. J Infect Dev Ctries 2014; 8(12): 1511-17.

18. Hussain M, Munir S, Fatima M, Rahim K, Ahmed I, Basit A, et al. Antimicrobial susceptibility patterns and CTX-M $\beta$-lactamase producing clinical isolates from burn patients in Islamabad, Pakistan. Asian Pac J Trop Dis 2017; 7(8): 486-90. 
19. Hatami R. The frequency of multidrug-resistance and extensively drug-resistant Acinetobacter baumannii in west of Iran. J Clin Microbiol Infect Dis 2018; 1(1): 4-8.

20. Lima WG, Alves GC, Sanches C, Fernandes SO, de Paiva MC. Carbapenem-resistant Acinetobacter baumannii in patients with burn injury: A systematic review and meta-analysis. Burns 2019 45(7): 1495-08.

21. Oberoi L, Singh N, Sharma P, Aggarwal A. ESBL, MBL and Ampc $\beta$ lactamases producing superbugs-Havoc in the Intensive Care Units of Punjab India. J Clin Diagn Res 2013; 7(1): 70-74.

22. Saaiq M, Ahmad S, Zaib MS. Burn wound infections and antibiotic susceptibility patterns at Pakistan Institute of Medical
Sciences, Islamabad, Pakistan. World J Plast Surg 2015; 4(1): 9-12. 23. Safaei HG, Moghim S, Isfahani BN, Fazeli H, Poursina F, Yadegari $S$, et al. Distribution of the strains of multidrug-resistant, extensively drug-resistant, and pandrug-resistant Pseudomonas aeruginosa isolates from burn patients. Adv Biomed Res 2017; 6(1): 74-78

24. McKinnell JA, Dwyer JP, Talbot GH, Connolly LE, Friedland I, Smith A, et al. Plazomicin for infections caused by carbapenemresistant Enterobacteriaceae. New Eng J Med 2019; 380(8): 791-93.

25. Lin WC. Emergence of carbapenem resistance in pseudomonas aeruginosa isolates from a specialized care facility for prolonged mechanical ventilation patients. Int J Inf Dis. 2019 79:47. 